\title{
Mid-infrared lasers for energy frontier plasma accelerators
}

\author{
I. V. Pogorelsky, ${ }^{1}$ M. N. Polyanskiy, ${ }^{1}$ and W. D. Kimura ${ }^{2}$ \\ ${ }^{1}$ Accelerator Tests Facility, Brookhaven National Laboratory, Upton, New York 11973 USA \\ ${ }^{2}$ STI Optronics, Inc., Redmond, Washington 98052 USA \\ (Received 10 May 2016; published 12 September 2016)
}

\begin{abstract}
Plasma wake field accelerators driven with solid-state near-IR lasers have been considered as an alternative to conventional rf accelerators for next-generation $\mathrm{TeV}$-class lepton colliders. Here, we extend this study to the mid-IR spectral domain covered by $\mathrm{CO}_{2}$ lasers. We conclude that the increase in the laser driver wavelength favors the regime of laser wake field acceleration with a low plasma density and high electric charge. This regime is the most beneficial for gamma colliders to be converted from lepton colliders via inverse Compton scattering. Selecting a laser wavelength to drive a Compton gamma source is essential for the design of such a machine. The revealed benefits from spectral diversification of laser drivers for future colliders and off-spring applications validate ongoing efforts in advancing the ultrafast $\mathrm{CO}_{2}$ laser technology.
\end{abstract}

DOI: 10.1103/PhysRevAccelBeams.19.091001

\section{INTRODUCTION}

Recent fundamental discoveries made at LHC further prove the importance of sustaining the ascent to highparticle-energy frontiers in order to gain deeper insight into matter and the Universe. To maintain this momentum, next-generation higher-energy accelerators are being contemplated by the global scientific community. TeV-class electron-positron $\left(e^{-} e^{+}\right)$linear colliders are among the most promising options for the next energy-frontier accelerator. Such a machine may provide the capability to investigate new physics beyond the standard model by discovering and exploring new particles and precisely measuring the Higgs boson and top quark beyond the LHC's reach.

Built on conventional rf technology, a linear collider would be of the order of $30 \mathrm{~km}$ long. To mitigate the considerable financial burdens imposed by such a project, scientists look to more economical and compact approaches that may be offered by replacing rf accelerators with plasma waves and high-power lasers.

An electron plasma wake, generated by particle bunches or laser pulses as they propagate through a plasma creates a strong longitudinal accelerating field $E_{0}[\mathrm{~V} / \mathrm{cm}] \approx$ $\sqrt{n_{e}\left[\mathrm{~cm}^{-3}\right]}$, e. g., $\sim 1 \mathrm{GeV} / \mathrm{cm}$ at a plasma density $n_{e}=10^{18} \mathrm{~cm}^{-3}$. Trapped in such a wake, electrons pick up several GeVs of kinetic energy from a palm-size $(1-10 \mathrm{~cm})$ accelerating stage. Recent demonstrations of such high-gradient electron acceleration, combined with good control over the properties of the accelerated beam, encourage researchers to contemplate plasma accelerators

Published by the American Physical Society under the terms of the Creative Commons Attribution 3.0 License. Further distribution of this work must maintain attribution to the author $(s)$ and the published article's title, journal citation, and DOI. up to $\mathrm{TeV}$ energies. While two methods of exciting wake fields in plasma using charged particles or laser pulses are deemed promising [1,2], we limit our analysis to Laser Wakefield Accelerators (LWFA) $[3,4]$.

In a number of publications and workshops, a roadmap toward $0.5-1.0 \mathrm{TeV} e^{-} e^{+}$colliders based on the LWFA method has been explored [5-8]. Capitalizing on the resounding success of LWFA experiments achieved by utilizing solid state lasers based on chirped pulse amplification (CPA) [9,10], the leading trend in designing prospective laser-plasma colliders concentrates on the near-IR spectral domain where these lasers operate.

While possible solutions for scaling plasma accelerators via multistaging look relatively transparent and are being poised for further testing, capabilities of present-day solidstate lasers measured by their average power, repetition rate, and energy efficiency are yet far below the numbers required to achieve the targeted collider performance at an acceptable operational cost. Efforts are being actively pursued to address these limitations of solid-state lasers, among which high-power fiber lasers appear particularly promising. While resolving these technical issues is still in the distant future, parallel evaluation of alternative laser technologies may be prudent from the standpoint of both revealing their technical capabilities and identifying new plasma acceleration regimes.

One of these technologies could be a $\mathrm{CO}_{2}$ laser with its distinctive radiation spectrum $(9-10 \mu \mathrm{m})$ fitting in the midIR range. After first pioneering demonstrations of plasma beat-wave acceleration [11] with the maximum $38 \mathrm{MeV}$ electron acceleration achieved [12], picosecond $\mathrm{CO}_{2}$ lasers surrender their competitive edge in the LWFA research to solid-state CPA lasers, femtosecond pulses of which resonantly drive plasma wakes for practically interesting plasma densities $n_{e} \geq 10^{16} \mathrm{~cm}^{-3}$. This situation will 
change with development of a new breed of ultrafast high-power $\mathrm{CO}_{2}$ lasers. The first laser of this generation is under construction at the BNL Accelerator Test Facility (ATF). This development prompts our outlook into the potentials of this emerging technology as a complementary path toward practically meaningful LWFA accelerators, TeV-class colliders in particular.

The stage for this discussion is set by a series of publications that elaborates on the LWFA collider concept built on near-IR laser drivers $[8,13,14]$. The purpose of this paper is to extend the scope of optimizing the laser parameters for plasma colliders into the mid-IR spectral domain covered by $\mathrm{CO}_{2}$ lasers. In order to make this study informative and self-consistent, we shall view the problem at several different angles, thereby shedding light on the underlying physics of the mid-IR LWFA process, its relevance to future colliders, and on the enabling capabilities of ultrafast $\mathrm{CO}_{2}$ lasers.

Section II discusses the specifics of using mid-IR laser drivers for LWFA. Our main purpose is to provide a comparative analysis of representative LWFA regimes driven by near-IR Ti:Sapphire (Ti:S) and mid-IR $\mathrm{CO}_{2}$ lasers for realizing a $1-\mathrm{TeV}$ center-of-mass (c. m.) collider with a targeted luminosity

$$
\mathcal{L}=\frac{f N_{e}^{2}}{4 \pi \sigma_{\perp}^{2}}=10^{34} \mathrm{~s}^{-1} \mathrm{~cm}^{-2},
$$

where $N_{e}$ is the number of particles per bunch, $f$ is the bunches' repetition rate, and $\sigma_{\perp}$ is the beam size at the collider interaction point (IP).

The analytical approach presented here for optimizing the plasma and laser parameters to achieve the required collider's luminosity, assuming feasible mid-IR laser parameters, is fully consistent with the formalism adopted in papers on near-IR LWFA colliders $[8,13]$, thus, permitting direct comparison of these two regimes. The reported earlier particle-in-cell (PIC) simulations for a single plasma accelerator stage driven with a prospective multi-terawatt, femtosecond $\mathrm{CO}_{2}$ laser [15] agree with our analytical predictions adding confidence to our analysis. We conclude that the optimum LWFA regime for $\mathrm{CO}_{2}$ lasers shifts towards lower plasma densities compared to Ti:S lasers. This introduces certain advantages, particularly related to a stronger bunch charge supported by a plasma wake. Entered quadratically into Eq. (1), a higher charge allows reducing the pulse repetition rate leading to lower power consumption by a collider.

We will see, however, that this positive trend is constrained by the beamstrahlung effect that puts a limit on a bunch charge. Nevertheless, there are other applications for mid-IR LWFA available, such as $\gamma \gamma$ colliders where beamstrahlung is not considered an issue. Converting lepton colliders into gamma colliders via inverse Compton scattering opens an opportunity to study a broader variety of processes by colliding $e^{-}, e^{+}$and $\gamma$ beams in any combination and at independently controlled polarization. Selecting the optimum laser to drive the Compton scattering is essential for the design of such a machine. Section III builds upon our analysis of selecting a $\mathrm{CO}_{2}$ laser as a Compton source driver for a 4.4-TeV c. m. $\gamma \gamma$ collider and for a Compton-based polarized positron source.

In Section IV, we discuss our strategy for developing the next-generation of ultrafast $\mathrm{CO}_{2}$ lasers that includes an isotopically enriched active laser medium, the chirped pulse amplification (CPA) technique, and nonlinear femtosecond compression. We also propose an approach for enhancing repetition-rate of $\mathrm{CO}_{2}$ laser amplifiers to satisfy the laser parameters targeted in Secs. II and III.

Resolving critical issues pertinent to ultrafast mid-IR laser technology can be viewed as milestones on a roadmap towards $\gamma \gamma$ colliders driven by next-generation $\mathrm{CO}_{2}$ lasers. Progress along this route will concurrently benefit other laser uses such as plasma-based compact synchrotron- and $\mathrm{THz}$ - radiation sources, laser-driven ion accelerators, and many more applications.

\section{MID-IR LWFA REGIMES OF RELEVANCE TO FUTURE LEPTON COLLIDERS}

The International Linear Collider (ILC), one of the leading candidates for the next-generation energy-frontier particle accelerator, will bring electron and positron beams into $1 \mathrm{TeV}$ c.m. collisions [16,17]. Such a machine might be near the limit of what can be constructed using conventional rf accelerator technologies, given reasonable space and cost constraints. One could envision a plasma-based $\mathrm{TeV} e^{-} e^{+}$ collider by coupling many LWFA acceleration stages together. Such a machine might require much smaller real estate being less expensive to build and operate.

Our comparative analysis of acceleration regimes relevant to multi-staged $1 \mathrm{TeV}$ colliders follows prior publications that prioritized LWFA driven with $\lambda=0.8 \mu \mathrm{m}$ Ti: $S$ laser drivers $[8,13]$. Although $\lambda$-scaling has been discussed for LWFA colliders $[8,18]$, it was not completed in a similarly comprehensive format as done for $\lambda=0.8 \mu \mathrm{m}$. Filling this gap, we elaborate here on $\lambda$-scaling of the LWFA collider regimes towards a mid-IR $\mathrm{CO}_{2}$ laser.

In Table I, we amend representative parameters for a collider driven by a Ti:S near-IR laser by adding parameter sets for a $\mathrm{CO}_{2}$ laser to illustrate that both these regimes fall into quite different parameter ranges. We note that selection of the $\mathrm{CO}_{2}$ carrier wavelength at $\lambda=9.2 \mu \mathrm{m}$ as opposed to traditionally used $\lambda=10.6 \mu \mathrm{m}$ is defined by utilizing an isotopic $\mathrm{CO}_{2}$ gas that shifts the maximum gain as will be discussed in Sec. IV.

We follow the approach to tabulating the collider's parameters developed in [8], which deals with a quasilinear LWFA regime that arguably provides better control over phasing, accelerating and focusing field components in a plasma wake suitable to accelerate both electrons and 
TABLE I. Representative examples of laser- and plasma-parameters for LWFA 1-TeV c.m. $e^{-} e^{+}$collider.

\begin{tabular}{|c|c|c|c|c|c|c|c|}
\hline Parameter & Ex1 & Ex2 & Ex3 & $\mathrm{Ex} 4$ & Ex 5 & $\mathrm{PIC}^{*}$ & Ex6 \\
\hline Laser wavelength $(\mu \mathrm{m})$ & 0.8 & 9.2 & 9.2 & 0.8 & 9.2 & 10 & 9.2 \\
\hline Plasma density $\left(\times 10^{16} \mathrm{~cm}^{-3}\right)$ & 11 & 11 & 1.1 & 1.1 & 0.35 & 0.32 & 0.11 \\
\hline Plasma wavelength $(\mu \mathrm{m})$ & 99 & 99 & 313 & 313 & 560 & 585 & 990 \\
\hline Laser pulse duration (fs) & 130 & 130 & 390 & 390 & 700 & 400 & 1300 \\
\hline Laser radius $(\mu \mathrm{m})$ & 63 & 63 & 200 & 200 & 360 & 262 & 630 \\
\hline Laser peak power (TW) & 300 & 2.3 & 23 & 3000 & 75 & 60 & 230 \\
\hline Laser energy per stage $(\mathrm{J})$ & 40 & 0.3 & 9 & 1200 & 52 & 24 & 300 \\
\hline Electrons per bunch $\left(\times 10^{9}\right)$ & 4 & 4 & 13 & 13 & 23 & 12 & 40 \\
\hline Single stage interaction length (m) & 0.79 & 0.06 & 0.19 & 25 & 1.1 & 0.6 & 6 \\
\hline Accelerating field $(\mathrm{GeV} / \mathrm{m})$ & 12.6 & 12.6 & 4.3 & 4.3 & 2.2 & 3.8 & 1.25 \\
\hline Energy gain per stage $(\mathrm{GeV})$ & 10 & 0.075 & 0.75 & 100 & 2.4 & 2.3 & 7.5 \\
\hline Number of stages & 50 & 6666 & 666 & 5 & 208 & 217 & 66 \\
\hline Collision rate $(\mathrm{kHz})$ & 10 & 10 & 1 & 1 & 0.3 & 1.2 & 0.1 \\
\hline Average laser power per stage $(\mathrm{kW})$ & 400 & 3 & 9 & 1200 & 16 & 30 & 30 \\
\hline Linac length (stages $0.5 \mathrm{~m}$ apart) (m) & 65 & 3300 & 466 & 125 & 448 & 240 & 430 \\
\hline Total laser power (MW) & 20 & 20 & 6 & 6 & 2.6 & 6.5 & 2 \\
\hline
\end{tabular}

${ }^{*}[15] ; a_{0}=2$ (for all other examples, $a_{0}=1.5$ ).

positrons. To satisfy this regime, we chose the normalized field strength parameter $a_{o}=e E / m \omega c=1.5$ at a laser peak power slightly exceeding the self-focusing threshold $P / P_{c r}=1.1$, where $P_{c r}=17\left(\frac{\omega}{\omega_{p}}\right)^{2}[\mathrm{GW}], \omega=2 \pi c / \lambda$ is the laser frequency, $E$ is the laser's electric field, $e$ and $m$ are, correspondingly, the electron charge and mass, $c$ is the speed of light, $\omega_{p}=\left(\frac{4 \pi e^{2} n_{e}}{m}\right)^{1 / 2}$ is the plasma frequency. Keeping the normalized LWFA parameters $a_{0}, k_{p} \times R$ and $\omega_{p} \times \tau$ fixed, where $k_{p}=\omega_{p} / c$ and $\tau$ is the laser pulse length the acceleration gradient scales as $E_{a} \propto n_{e}^{1 / 2}$, the number of electrons per bunch $N_{e} \propto n_{e}^{-1 / 2}$, and $P \propto \lambda^{-2} n_{e}^{-1}$. These simple scaling rules [8] lead to defining the entries to Table I for different selections of $\lambda$ and $n_{e}$.

It is assumed that electron dephasing can be compensated by proper tapering of the plasma density within a single accelerating stage. Therefore, the length of an individual stage is limited by the laser depletion. As the laser energy is depleted within a laser-plasma accelerating stage, a fresh laser pulse must be coupled into the next stage. We assume $50 \mathrm{~cm}$ for a coupling distance between stages to provide for laser injection and e-beam manipulation. The number of stages is adjusted to achieve $0.5 \mathrm{TeV}$ energy, in a single beam. The repetition rate $f$ is chosen to achieve the required luminosity $\mathcal{L}=10^{34} \mathrm{~s}^{-1} \mathrm{~cm}^{-2}$ with assumed $\sigma_{\perp}=10 \mathrm{~nm}$.

We begin Table I with Example 1 for an optimum set of $0.8-\mu \mathrm{m}$ collider parameters from [8]. Example 2 shows a $\mathrm{CO}_{2}$ laser reaching a quasilinear LWFA regime for the same $10^{17} \mathrm{~cm}^{-3}$ plasma density, at 100 times smaller laser power and energy as a direct result from the laser wavelength scaling of an electron's ponderomotive potential $\Phi_{p}=\frac{e^{2} E^{2}}{4 m \omega^{2}}$. However, a very short acceleration stage defined by the laser depletion distance, $L_{d}=\lambda_{p}^{3} / \lambda^{2}$, where $\lambda_{p}$ is the plasma wavelength, results in a small energy gain per stage and a huge number of stages required to reach the
$0.5 \mathrm{TeV}$ particle energy. The total multi-staged accelerator length will be largely affected in this case by the coupling spaces between stages.

The tenfold reduction in the plasma density quoted in Example 3 results in a longer depletion (accelerating stage) length, thereby allowing a proportional increase in net acceleration per stage, thus, reducing the total accelerator length. A higher accelerated charge permits reducing the repetition rate and relaxing the cumulative average laser power. Note that operating in such a low-density regime with a 0.8- $\mu \mathrm{m}$ laser, as shown in Example 4, results in extremely high values for the peak- and average-power of the individual drive lasers and the need to maintain acceleration over $25 \mathrm{~m}$ of stage length, which are not deemed practical nor feasible.

The benefit of reducing the plasma density for a $\mathrm{CO}_{2}$ laser, as unveiled by Example 3, points toward the possibility for further optimizing the parameter map for a collider with a mid-IR laser driver. We, therefore, calculated two more Examples 5 and 6 with a finer coverage of the low plasma density range. These examples show that further dropping the plasma density reduces the number of accelerating stages and cumulative average power of the laser drivers. However, individual laser parameters become more challenging to achieve.

Finally, we compare Examples 1 and 5 that represent nearly optimum parameter sets for colliders driven by the $0.8-\mu \mathrm{m}$ and $9.2-\mu \mathrm{m}$ lasers, respectively. For both cases, the length of an accelerating stage is about the same, $\sim 1 \mathrm{~m}$. The net acceleration per stage is higher for Ti:S (Example 1), which results in fewer stages and a shorter linac. However, individual and cumulative power requirements for the $\mathrm{CO}_{2}$ laser case (Example 5) are tremendously relaxed, which is a direct consequence of the increase in the bunch charge at lower plasma densities. 
The validity of our analytical estimates for the $\mathrm{CO}_{2}$ laser case is confirmed by benchmarking with PIC simulations [15], entered into Table I. In spite of small differences in input conditions, the simulated results are well within ballpark agreement with the analytical estimates in Example 5. The 2-nC charge per bunch assumed in the simulations is quite arbitrary and represents a conservative assumption for the amount of charge that favors preserving good beam quality. This reduced charge calls for a higher repetition rate and more stringent requirements on the laser's energetics than presented in the analytical Example 5. Otherwise, the agreement in terms of the energy gain per stage is quite reassuring.

Thus far we have considered the maximum accelerated charge per bunch at a quarter of the beam-loading limit for a blow-out regime defined by [19],

$$
N_{\mathrm{e}} \approx 1.1 \times 10^{9} \lambda[\mu \mathrm{m}] \sqrt{P[\mathrm{TW}]} .
$$

Maximizing the charge aids in satisfying the required collider luminosity at a reduced laser repetition rate and average power. However, the dominating limit for the bunch charge is imposed by particle beam degradation at the IP due to beam-beam interaction called beamstrahlung. This is parasitic gamma radiation that causes a background noise on detectors set around the IP, reduces the particle beam energy, $\mathcal{E}_{e}$, and elevates the beam energy spread. In general, the beamstrahlung must be kept below one gamma photon per lepton, and the induced $\delta \mathcal{E}_{e}$ - a few tens of percent. In our case, this requirement translates to limiting a number of particles per bunch, $N_{e} \approx 3 \times 10^{9} \quad$ [14]. Considering a rather shallow dependence of both the number of beamstrahlung photons and the induced beam energy spread upon the bunch charge $\propto N_{e}^{1 / 3}$, the severity of the beamstrahlung factor in setting the design limit on $N_{e}$ is yet to be seen. Meanwhile, in view of this concern, we proceed further under assumption that the low-density cases in Examples 3-6 could be of limited utility for $e^{-} e^{+}$colliders. Therefore, in the next Sec. III, we discuss gamma colliders where beamstrahlung is not a limiting factor.

\section{USE OF $\mathrm{CO}_{2}$ LASERS IN GAMMA COLLIDERS}

\section{A. Scientific case and technical feasibility for a $\gamma \gamma$ collider}

Lepton linear colliders can be readily converted to photon colliders by introducing inverse Compton backscattering. This offers a unique opportunity to study lepton- $\gamma$ and $\gamma \gamma$ interactions at comparable energies and luminosities in addition to $e^{-} e^{+}$collisions.

Although a $\gamma \gamma$ collider is presently not among the top choices for next frontier accelerators, it can offer additional scientific opportunities not to be overlooked.
The physics case for a high-energy photon collider has been thoroughly reviewed in [20]. Arguably the most interesting processes involve the direct production of a Higgs boson. Supersymmetric Higgs bosons can be produced in photonphoton collisions in energy regions not accessible with LHC, while control of polarization of the Compton photons will allow verification of the circular polarization nature of Higgs bosons.

Another example is charged pair-production, for instance $W^{+} W^{-}$or $t t$ pairs or other new supersymmetric particles. Cross sections for the production of charged scalar, lepton, and top pairs in $\gamma \gamma$ collisions are larger than those in $e^{-} e^{+}$collisions by a factor of $5-10$; for $W^{+} W^{-}$ production this factor is even larger, about 10-20. Therefore, the $\gamma \gamma$ collider is considered as a valuable extension to a lepton collider.

Furthermore, if a dedicated stand-alone $\gamma \gamma$ collider might ever be considered, one should have in mind its potential technical advantages such as: (a) eliminating the need for a more complex positron accelerator; two electron beams will suffice; (b) prospects of utilizing a more energyefficient and higher luminosity low-density plasma regime; $\mathrm{CO}_{2}$ lasers may have a special value in this context. As long as the beamstrahlung restriction on the maximum bunch charge is no longer applicable, scaling of the bunch particle number with plasma density $N_{e} \propto n_{e}^{-1 / 2}$ can be fully exploited. Considering that $\mathscr{L} \propto N_{e}^{2}$, this results in an opportunity to reduce $f \propto n_{e}$, as well as the electron beam power and the total average laser power $\propto n_{e}^{1 / 2}$. As we can see by examples from Table I, the increase in a depletion distance and acceleration per stage at reduced plasma density results in a proportional increase of the energy requirements for an individual laser. This trend is particularly stressful for near-IR lasers in low-density regimes, $n_{e} \leq 3 \times 10^{16} \mathrm{~cm}^{-3}$, calling for multi-PW peak power and multi-kJ pulse energy, whereas, mid-IR $\mathrm{CO}_{2}$ laser parameters appear more practical and accessible (with their tens of TW and tens of Joule for the same beam charge).

\section{B. $\mathrm{CO}_{2}$ laser as a Compton driver for $\gamma \gamma$ collider}

The analysis presented in this section follows the footsteps of earlier publications that address laser requirements for controlling the electron-to-photon Compton conversion in gamma colliders [21-23].

When designing the optimum configuration and parameters for a Compton IP, several laser parameters are important; laser wavelength is one of them. The energy of Compton photons depends upon both the particle energy, $\mathcal{E}_{e}$, and laser frequency, $\omega$, according to

$$
\omega_{\gamma}=\frac{x}{x+1} \frac{\mathcal{E}_{e}}{\hbar},
$$

where $x=4 \mathcal{E}_{e} \hbar \omega / m^{2} c^{4}, \quad \hbar$ is the Plank constant. According to Eq. (3), the condition for efficient conversion 
of particle energy to photon energy is $x \gg 1$; this suggests choosing the laser wavelength to be as short as possible. However, a strong limitation to the laser wavelength is imposed by rescattering of Compton photons by the laser beam into $e^{-} e^{+}$pairs. This occurs when the product of the laser photon energy, $\hbar \omega$, and the gamma energy, $\hbar \omega_{\gamma}$, is greater than the square of the electron rest energy, that is when $\omega \omega_{\gamma}>m^{2} c^{4} / \hbar^{2}$. Based on this condition and using Eq. (3), the optimum laser wavelength is derived: $\lambda[\mu \mathrm{m}]=4.2 \mathcal{E}_{e}[\mathrm{TeV}]$, with corresponding $x=4.8$ and $\hbar \omega_{\gamma}=0.83 \mathcal{E}_{e}$. For example, for the $\mathcal{E}_{e}=0.5 \mathrm{TeV}$ case, a laser with $\lambda=2 \mu \mathrm{m}$ is required, which changes to $\lambda=9.2 \mu \mathrm{m}$ for $\mathcal{E}_{e}=2.2 \mathrm{TeV}$.

Other important parameters for a laser driver are power, pulse duration, $\tau$, and focusing of the laser beam. The main consideration when choosing the laser focusing is to make it comparable with the e-beam dimensions within the Compton interaction distance to ensure efficient utilization of both beams. The smallest laser focus spot size $R$ is related to $\tau$, when the laser pulse length fits to the Rayleigh length

$$
R^{2} \geq \frac{c \tau \lambda}{2 \pi^{2}}
$$

The laser intensity needs to be sufficiently high to ensure the efficient conversion of the e-beam energy into gamma photons. The probability of this conversion, $p$, is the product of the laser photon density,

$$
n_{L}=E_{L} / \hbar \omega \pi R^{2} \tau c
$$

Compton scattering cross section, $\sigma_{C} \approx \frac{\pi r_{e}^{2}(2 \ln x+1)}{x}$ $\left(=1.9 \times 10^{-25} \mathrm{~cm}^{2}\right.$ at $\left.x=4.8\right)$, and the interaction length along the electron beam propagation are defined by the laser pulse duration:

$$
p=n_{L} \sigma_{C} \tau c .
$$

Substitution of Eq. (5) into Eq. (6) gives $n_{L}=E_{L} / \hbar \tau^{2} c^{3}$. And assuming $p=1$, we arrive at the required laser power $P \equiv E_{L} / \tau=\hbar c^{2} / \sigma_{C} \cong 1 \mathrm{TW}$, which is invariant to $\lambda$.

Note that $\tau$ is not a free parameter and can be further defined assuming $a_{0}<1$, which is the condition for the linear Compton scattering. By expressing the laser electric field through the laser peak power, $P$, and radius, $R$, and relating this to the pulse duration through Eq. (4), one obtains $\tau[\mathrm{ps}] \approx \frac{0.7 \lambda[\mu \mathrm{m}]}{a_{0}^{2}}$. Taking $a_{0}^{2}=0.1$, we conclude that the optimized conversion of $\mathcal{E}_{e}=2.2 \mathrm{TeV}$ electron beams to gammas calls for a $\mathrm{CO}_{2}$ laser with $\tau \approx 70 \mathrm{ps}$ and $E_{L} \approx 70 \mathrm{~J}$. The resulting photon beam energy is $\sim 1.8 \mathrm{TeV}$ and photon collider luminosity is comparable with that defined by the particle beams.

\section{C. $\mathrm{CO}_{2}$ laser as a positron source for $\boldsymbol{e}^{-} \boldsymbol{e}^{+}$collider}

Regardless of the method of accelerating particles in a future $e^{-} e^{+}$collider, whether it is a conventional $\mathrm{rf}$ or advanced plasma accelerator, high-flux production of polarized positrons is another area where lasers may find their application.

Intense beams of circularly polarized gamma rays in the $30 \mathrm{MeV}$ energy range are required for producing polarized positrons for the next-generation $e^{-} e^{+}$linear colliders, such as the ILC and the Compact Linear Collider (CLIC) [24]. Two basic schemes for a polarized positron source (PPS) are being considered for the ILC: spontaneous radiation of a $150-\mathrm{GeV}$ electron beam in a 200 -meter-long helical wiggler $[25,26]$, and Compton backscattering of a highintensity laser beam from a $1.3-4 \mathrm{GeV} e$-beam [27,28].

PPS based on Compton backscattering (CPPS) is relatively compact, independent from the main linac, and can offer considerable flexibility, such as easy switching of positron polarization, which is determined by the laser. Several key principles for designing a Compton source for positron production echo similar requirements pertinent to a $\gamma \gamma$ collider, as discussed above. Some of these conditions are a close overlap between counterpropagating electron and laser pulses, and the laser power required to achieve a high conversion factor $N_{\gamma} / N_{e} \approx 1$ in the linear scattering regime. The most notable difference compared to the requirements for the Compton IP of $\gamma \gamma$ colliders is that the scattering process for CPPS is actually in the Thomson regime characterized by insignificant electron energy depletion. This allows each electron to participate multiple times in the gamma production. Furthermore, a low efficiency of gamma conversion to positrons on a target $(\sim 2 \%)$ requires a multi-kHz laser pulse repetition rate that can be achieved using a laser pulse circulating inside a closed-loop optical cavity. The concept of an intra-cavity CPPS has been under investigation for several years [20,29-31]. One of the main distinctions between the different CPPS proposals is the wavelength of the laser. The choice typically varies from $\sim 0.8-1 \mu \mathrm{m}$ for solid-state lasers, to $\sim 10 \mu \mathrm{m}$ for $\mathrm{CO}_{2}$ lasers. The $\mathrm{CO}_{2}$ approach has been promoted in [31]. Pertinent to CPPS a higher Compton cross section $\sigma_{C}=6.65 \times 10^{-25} \mathrm{~cm}^{2}$, the possibility of "staking" $\gamma$-pulses over multiple consecutive interaction points, and pulse recirculation in a cavity result in considerably relaxed $\mathrm{CO}_{2}$ laser parameters, calling for just $2 \mathrm{~J}$ in $5 \mathrm{ps}$ and $0.75 \mathrm{~kW}$ average power.

\section{Summary of $\mathrm{CO}_{2}$ laser applications for Compton sources in future colliders}

Table II summarizes the two examples discussed here, $\mathrm{CO}_{2}$ laser applications as Compton drivers in future colliders including a gamma IP in the 4.4-TeV collider, and a CPPS. These distinctive areas of $\mathrm{CO}_{2}$ laser applications require different laser parameters ranging from more 
TABLE II. Design parameters of $\mathrm{CO}_{2}$ lasers for Compton applications in future colliders.

\begin{tabular}{lcc}
\hline \hline Parameter & $\gamma \mathrm{IP}$ & $e^{+}$source \\
\hline Electron energy & $2.2 \mathrm{TeV}$ & $4 \mathrm{GeV}$ \\
$\gamma$ energy & $1.8 \mathrm{TeV}$ & $40 \mathrm{MeV}$ \\
Laser pulse duration $(\mathrm{ps})$ & 70 & 5 \\
Laser peak power $(\mathrm{TW})$ & 1 & 0.4 \\
Laser energy per stage $(\mathrm{J})$ & 70 & 2 \\
Laser focus radius $(\mu \mathrm{m})$ & 200 & 70 \\
Laser repetition rate $(\mathrm{Hz})$ & $300(30)$ & 150 \\
Pulses in a train & $1(10)$ & 100 \\
Average laser power $(\mathrm{kW})$ & 20 & 1 \\
Number of lasers & 1 & 10 \\
\hline \hline
\end{tabular}

challenging for a $\gamma \gamma$ collider to more readily accessible in the case of a positron source for an $e^{-} e^{+}$collider.

The first example addresses the application of a $\mathrm{CO}_{2}$ laser for producing gamma rays via inverse Compton scattering for the final IP of the $4.4 \mathrm{TeV}$ c.m. collider, as discussed in Sec. III B. The required 70-ps long pulse can be achieved within limits of presently available laser technology. Numbers in brackets correspond to the scenario when ten identical laser pulses are produced during a single amplifier shot. Other resources for attaining high-repetition $\mathrm{CO}_{2}$ laser operation will be addressed in Sec. IV.

The second case of a polarized positron source for a linear collider is the least demanding. Note that we consider here a conventional rf linac with a pulse format significantly different from that assumed for laser plasma colliders. To match this pulse format, a pulsed laser operating at $150 \mathrm{~Hz}$ should support an intra-cavity circulation of 100 pulses per shot that results in a cumulative pulse repetition rate of $15 \mathrm{kHz}$. A recent demonstration of cumulative $\mathrm{x}$-ray flux multiplication, where the laser/e-beam IP has been set inside a regenerative $\mathrm{CO}_{2}$ amplifier cavity, has proven the viability of this concept [32].

\section{ROADMAP TO NEXT-GENERATION ULTRAFAST $\mathrm{CO}_{2}$ LASERS}

Our analysis presented in Sec. II reveals attractive opportunities from applying mid-IR laser beams for LWFA. Several examples compiled in Table I indicate the need for sub-picosecond $\mathrm{CO}_{2}$ pulses at multi-terawatt peak power-a regime not realized yet with $\mathrm{CO}_{2}$ lasers. Driven by this demand, a conceptual approach for achieving such parameters is being investigated at the ATF. The ATF presently hosts a 2-TW, picosecond $\mathrm{CO}_{2}$ laser in combination with a high-brightness $80-\mathrm{MeV}$ electron linac to support experimental programs in high-energy physics and other multi-disciplinary applications [33]. Achieving an ultrafast regime at ultra-high peak power requires novel methods never applied to molecular gas lasers until now. We unveil here this new approach and its implementation in our project BESTIA (Brookhaven Experimental SupraTerawatt Infrared at ATF) [34], recently initiated at ATF.

When developing a laser system to produce an ultrashort pulse comprising just a few light wave oscillations, the first question is: How to generate a short seed pulse? Ultrafast, high-power solid-state lasers use a mode-locked oscillator as a front end. This is not a solution for mid-IR gas lasers, which do not support this mode of operation due to their limited bandwidth, short inversion lifetime, and lack of electro-optical materials for mode-locking switches. Therefore, different indirect methods for generating mid-IR short pulses have been implemented based on using solidstate laser beams for fast modulating the optical properties of IR materials. This includes dynamic change of mid-IR reflectivity in superconductors and polarization rotation in birefringent liquids. These methods have been successfully utilized for selecting picosecond slices from $\mathrm{CO}_{2}$ laser beams [35-37].

Another source of ultrafast coherent pulses in the mid-IR is by conversion of near-IR (primarily Ti:S) laser radiation using nonlinear crystals in Optical Parametric Amplifiers (OPA). Although known for years, this method has never been implemented as a front-end for a high-power $\mathrm{CO}_{2}$ laser system until now, when an OPA has been set in operation for the ATF $\mathrm{CO}_{2}$ laser system [37]. A schematic diagram of the ATF's OPA front end is shown in Fig. 1. This commercial laser system (PALITRA, Quantronix Inc.) utilizes a frequency-doubled erbium oscillator and diodepumped Ti:S regenerative amplifier. A femtosecond parametric down-convertor uses nonlinear crystals to produce and then mix beams at $\lambda_{1}=1.5 \mu \mathrm{m}$ and $\lambda_{2}=1.7 \mu \mathrm{m}$ to generate a difference frequency signal ranged between

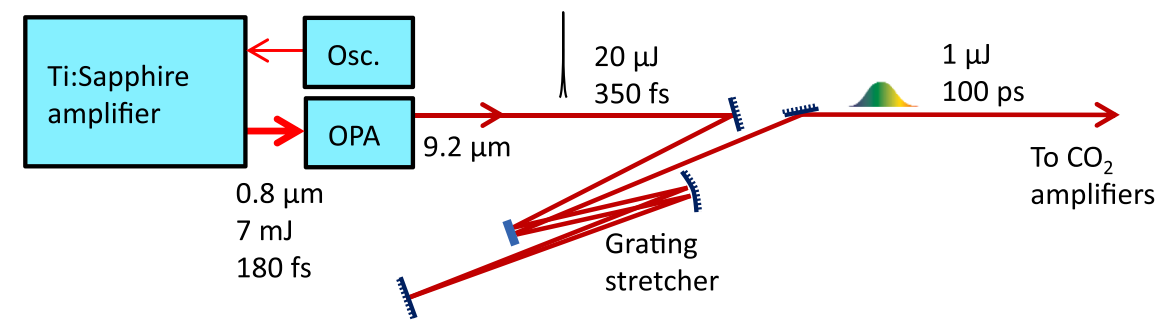

FIG. 1. BESTIA front end featuring a MOPA (Master Oscillator - Power Amplifier), the OPA seed pulse generator, and a CPA stretcher. 
8-11 $\mu \mathrm{m}$ with output parameters 350 fs and $20 \mu \mathrm{J}$ at a repetition $240 \mathrm{~Hz}$.

The overall bandwidth of the OPA output is much larger than the actual gain spectrum that can be amplified in the $\mathrm{CO}_{2}$ laser. Spectral filtering in the stretcher, which also serves for CPA, assures that the seed pulse matches a branch $(9 \mathrm{R})$ of the gain spectrum of the $\mathrm{CO}_{2}$ active medium.

A possible upgrade for the front-end of an ultrafast midIR laser system is foreseen by increasing the seed pulse energy. A stronger seed will permit simplifying the subsequent $\mathrm{CO}_{2}$ amplifier chain; reduce the pulse phase- and spectral- distortions upon amplification, and improving the pulse contrast. This increase in pulse energy beyond the limits of conventional OPA will be accomplished by adding another laser-pumped amplifier crystal. Pulse stretching before this crystal allows use of more energetic picosecond lasers for a pump and reduces nonlinear pulse distortions in the crystal. A chirped $(\sim 1 \mathrm{~mJ})$ pulse produced after such an upgraded OPCPA (Optical Parametric Chirped Pulse Amplifier) will be sent through $\mathrm{CO}_{2}$ amplifiers and then recompressed in a grating compressor.

High-pressure, 10-bar, isotopic $\mathrm{CO}_{2}$ laser amplifiers will be required to raise the seed pulse energy to the multi-Joule level $[38,39]$. The double action of the spectral pressure broadening and multi-isotope $\left({ }^{16} \mathrm{O}-{ }^{18} \mathrm{O}\right)$ mixing provides a bandwidth sufficient for amplification of 1.5-2 ps pulses with the maximum gain in the $\mathrm{CO}_{2}$ spectral branch centered at $9.2 \mu \mathrm{m}$. This regime has been tested already at the ATF on a smaller scale in the first operational isotopic picosecond $\mathrm{CO}_{2}$ laser amplifier [40], which will be also used for the first amplifier stage in BESTIA. This laser amplifier with an active volume $1 \times 1.5 \times 80 \mathrm{~cm}^{3}$ will operate in a regenerative regime where the pulse extraction out of the optical cavity is accomplished by a semiconductor $(\mathrm{Ge})$ optical switch controlled with a Nd:YAG laser pulse. This amplification stage will provide $\sim 10^{4}$ net energy gain achieved in multiple passes.

Our second, main amplifier is being built to accommodate three high-pressure gas-discharge sections, each having a $10 \times 10 \times 100 \mathrm{~cm}^{3}$ active volume. This design allows setting a multi-pass, folded beam propagation through individual amplifier sections culminated with a final swiping pass through the entire 3-meter active length to ensure efficient energy extraction and beam profile uniformity. The output energy from this amplifier should reach $70 \mathrm{~J}$, corresponding to a net energy gain $\sim 10^{4}$.

A CPA method will be implemented to mitigate nonlinear pulse distortions on the laser's optical components, where the Kerr effect on the aforementioned Ge optical switch and the amplifier's $10-\mathrm{cm}$ thick output $\mathrm{NaCl}$ window are of prime concern. Being an integral part of present-day ultraintense solid state laser systems, the CPA method has not been applied to gas lasers until recently when we demonstrated its advantages for improving performance of the ATF's regenerative amplifier [41]. Our stretching of a 1-ps pulse to $80 \mathrm{ps}$ improved, by an order of magnitude, the energy extraction from the regenerative amplifier via relieving nonlinear absorption and refraction on the amplifier's optical elements, an intracavity Ge wafer in particular. One of the questions to be answered by that study was: Will the residual rotational modulation in the molecular gas gain spectrum impede the recompression of the amplified $\mathrm{CO}_{2}$ laser pulse? By simulation and through experiment we have found that the CPA compression is barely affected by this spectral modulation. The duration of the pulse after a grating compressor was $1.6 \mathrm{ps}$, in good agreement with the pulse's measured spectral bandwidth. We consider this accomplishment as a pivotal step towards next-generation ultraintense $\mathrm{CO}_{2} \mathrm{CPA}$ laser systems. Implementing the CPA technique through the final stage of amplification in BESTIA should allow a more comfortable extraction of the $\sim 70-\mathrm{J}$ laser energy from the final amplifier. The pulse's recompression with diffraction gratings to $\sim 2 \mathrm{ps}$ should provide $\sim 25$ TW peak laser power (see Fig. 2).

For additional power increases, we plan on using a novel nonlinear femtosecond compressor [42]. When an intense optical pulse propagates through a medium, the refractive index of the latter evolves following the intensity profile of the pulse. A corresponding variation of the phase velocity results in the spectral broadening and chirping of the pulse. This effect is known as self-phase modulation, or selfchirping. For a Gaussian beam, self-phase modulation (SPM) is always accompanied by self-lensing, which is normally considered as unwelcome distortion of the beam's collimation. We plan to utilize this effect to our advantage by using a spatial filter to separate the high-intensity part of the pulse, where the spectral chirp is strong and close to

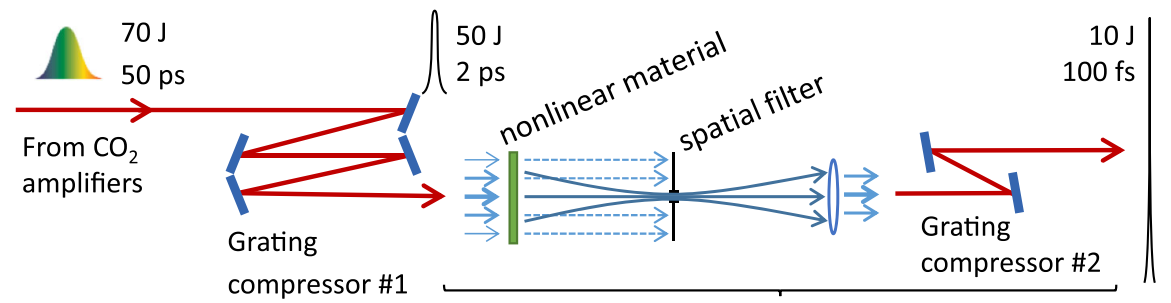

Nonlinear compressor

FIG. 2. The CPA compression followed by the nonlinear pulse compressor setup. 
linear, from the low-intensity pedestal. The transmitted beam is then collimated and further compressed in a second grating compressor as is illustrated in Fig. 2. The simulated output beam parameters (100 TW @ 100 fs) represent the case of an ideal Gaussian beam and high-efficiency grating compressor. Practical demonstration of these parameters is the subject of ongoing R\&D.

Fortunately, the collider requirements drafted in Secs. II and III offer some flexibility on the laser design parameters such as pulse duration and peak power (see Example 3). We expect that the BESTIA's single-shot performance will be within ballpark numbers for the LWFA and Compton IP drivers and will exceed requirements for a positron source.

While the peak laser parameters for the collider application seem within reach, the average power is lacking. The collider's requirements formulated in the previous sections call for $100-1000 \mathrm{~Hz}$ pulse repetition rate and on the order of $10 \mathrm{~kW}$ average power. Although short-pulse $10-\mathrm{kW} \mathrm{CO}$ lasers have been demonstrated before [43], this has been accomplished within a nanosecond pulse format that is technically quite different from the sub-picosecond regime required for the collider application. The BESTIA's front-end OPA pulse injector operates at $240 \mathrm{~Hz}$ already. Such OPAs and their improved OPCPA versions are also easily upgradable to $1 \mathrm{kHz}$ repetition rate. $\mathrm{CO}_{2}$ lasers are commercially available with comparable high repetition rate and long life performance. Of course, these high repetition rate $\mathrm{CO}_{2}$ laser designs are not at the multi-TW level needed for a future collider but could be utilized for smaller-scale versions of high-pressure lasers similar to the one used for a regenerative amplifier in BESTIA.

Constructing a high-rep-rate, high-power $\mathrm{CO}_{2}$ laser amplifier requires addressing several engineering issues. The most severe of them is making a high-voltage (HV) pulsed power circuit to deliver the energy to the electric discharge at a high repetition rate. For a multi-TW amplifier, the gas pressure is high $(10 \mathrm{~atm})$. The high output energy rating of this laser means that its aperture must be large to accommodate laser beams with the large transverse sizes to avoid damaging the amplifier's window. Hence, the interelectrode gap in such an amplifier is also large (10 cm for BESTIA amplifier). The high-pressure and a large gap mean that the HV discharge should operate at $\geq 600 \mathrm{kV}$ and peak currents $\geq 40 \mathrm{kA}$ as is required to deposit sufficient energy in the laser gas to achieve a specific gain of $2.0 \% / \mathrm{cm}$. In addition, in order to sustain a high-pressure and uniform gas discharge, the current rise time should not be longer than 100-200 ns. HV drivers at such voltages and currents typically employ Marx banks with spark gaps used for HV switches as in the case of the BESTIA laser system, which will be limited to sub-Hz repetition rate primarily by the spark-gaps. Although there is no known direct prototype for a high-repetition-rate, long-lifetime HV driver that satisfies our requirements, we anticipate that a solution could be sought on the way of replacing spark gaps with thyristors known to operate up to $350 \mathrm{~Hz}[44,45]$. Their primary shortcoming is their inherently long current rise time, $\sim 500$ ns. However, this can be mitigated by implementing magnetic pulse compression, which is a standard technique for increasing the peak current and reducing rise time of the pulse using saturable inductors [46]. Compression factors of over 5 times have been demonstrated for projects associated with excimer laser development [47]. This implies that the circuit rise-time can be reduced from $\sim 500 \mathrm{~ns}$ to $\sim 100 \mathrm{~ns}$ while the 10-kA theristor's peak current can be simultaneously increased to $\sim 50 \mathrm{kA}$, as is required for a TW-class laser.

Based on the presented analysis, the following roadmap on the way to developing enabling $\mathrm{CO}_{2}$ laser technologies and applications relevant to the next-generation collider concepts can be envisioned: Presently available $2-3$ ps mid-IR laser pulses can be readily amplified to the multi-terawatt peak power to enable proof-of-principle self-modulated LWFA experiments in the previously unexplored spectral domain, as well as inverse Compton scattering studies of the gamma-collider relevance.

Shorter, $0.5-1 \mathrm{ps}, \mathrm{CO}_{2}$ laser pulses are very likely to be demonstrated within the next two-three years to allow further explorations into low-plasma-density quasilinear and "bubble" LWFA regimes, their external seeding and staging important for the LWFA collider design.

Still shorter, 0.3-0.1 ps, pulses could be the subject of a more extensive and higher-risk laser R\&D that might continue on the scale of the next 5-10 years alongside with developing high-repetition versions of such lasers toward their practical applications beyond the fundamental physics and proof-of-principle demonstrations.

\section{CONCLUSIONS}

The vision of replacing conventional rf accelerator technology with compact laser plasma accelerators promises an economical alternative to attaining $\mathrm{TeV}$-class energy frontiers of interest for next-generation $e^{-} e^{+}$colliders and beyond. In this paper, we analyzed prospects for developing future accelerators and colliders on the platform of midIR $\mathrm{CO}_{2}$ lasers. We identified critical issues in selecting $\mathrm{CO}_{2}$ lasers for these applications and compared performance of such prospective laser-plasma accelerators with ones based on near-IR solid-state lasers.

The underlying physics of plasma wakes points to the process optimization where the plasma wavelength changes in accord with a laser wavelength. A high-captured bunch charge is one of the advantages of a low-density LWFA regime prioritized for $\mathrm{CO}_{2}$ laser drivers. However, this highcurrent regime may have a limited utility for lepton colliders due to parasitic beamstrahlung losses from beam-beam interaction at the IP. This restriction is not applicable to $\gamma \gamma$ colliders where the beamstrahlung is not an issue. Notably, $\mathrm{CO}_{2}$ lasers can be considered for $\gamma \gamma$ collider projects not just as drivers for LWFA stages, but could be 
a unique fit for a Compton source where the electron beam energy is converted to gamma rays. $\mathrm{CO}_{2}$ lasers can also be used for driving a Compton polarized positron source in a lepton collider, including its more conventional rf alternative.

We reviewed options for achieving the laser peak parameters required for the aforementioned applications and addressed ways for attaining high repetition rates of laser pulse delivery compatible with the collider's pulse format.

Ongoing development of ultrafast $\mathrm{CO}_{2}$ laser technology opens, for the first time, opportunities for exploring mid-IR LWFA regimes pertinent to colliders. Notably, development of a new category of $\mathrm{CO}_{2}$ lasers able to drive future colliders simultaneously supports many other important applications that further justify this effort. Indeed, the utility of mid-IR lasers for advanced accelerator research is much broader than just being a dedicated driver the future collider: It can be regarded as a research tool to enable new opportunities for explorations in LWFA physics, including studies of wakefields and plasma density distributions in plasma wakes, bunch seeding, and accelerator staging. All of these features and processes are easier to investigate within the larger plasma cavities created by longer-wavelength $\mathrm{CO}_{2}$ lasers.

Oversized plasma bubbles to be readily produced by $\mathrm{CO}_{2}$ lasers will sustain high-current electron bunches. The same feature, combined with localized injecting of seed electrons by means of external laser ionization in two-color LWFA scheme [48,49], might support acceleration of low-emittance electron bunches. This capability of a low-density LWFA combined with the proportional to $\lambda$ copious number of photons per energy unit from another counter-propagating $\mathrm{CO}_{2}$ laser pulse, promises the highest gamma- or x-ray yield in inverse Compton scattering considered for compact light sources.

Examples of potential applications of high-repetition, ultrafast $\mathrm{CO}_{2}$ lasers can be continued beyond LWFA, including ion acceleration from over-critical plasmas, plasma-based $\mathrm{THz}$ radiation sources, and more $[50,15]$.

\section{ACKNOWLEDGMENTS}

The authors wish to thank I. Ben-Zvi, M. Palmer, M. Babzien and J. Skaritka (BNL) for their contribution to ongoing development of the next-generation, ultrafast $\mathrm{CO}_{2}$ laser BESTIA and W. Lu (Tsinghua Univ.) for useful discussions. This work is supported by the US DOE Contract No. DE-AC02-98CH10886, and STI Optronics Internal Research and Development funding. A partial funding of the BESTIA project comes from the BNL Facility Development Grant No. PD14-009.

[1] C. Joshi and T. Katsouleas, Plasma accelerators at the energy frontier and on tabletops, Phys. Today 56, No. 6, 47 (2003).

[2] C. Joshi, Plasma accelerators, Sci. Am. 294, 41 (2006).
[3] T. Tajima and J. M. Dawson, Laser Electron Accelerator, Phys. Rev. Lett. 43, 267 (1979).

[4] S. P. D. Mangles et al., Monoenergetic beams of relativistic electrons from intense laser-plasma interactions, Nature (London) 431, 535 (2004).

[5] M. Xie, T. Tajima, K. Yokoya, and S. Chattopadhyay, Advanced accelerator development strategy report, Report of DOE Advanced Accelerator Concepts Research Roadmap Workshop, 2016, http://science.energy.gov/ / media/hep/pdf/accelerator-rd-stewardship/Advanced_ Accelerator_Development_Strategy_Report.pdf.

[6] W. Leemans and E. Esarey, Laser-driven plasma-wave electron accelerators, Phys. Today 62, No. 3, 44 (2009).

[7] B. Foster, Golden state of linear colliders, http://newsline .linearcollider.org/2016/01/14/the-goldenstate-of-linear-colliders/.

[8] C. B. Schroeder, E. Esarey, C. G. R. Geddes, C. Benedetti, and W. P. Leemans, Physics considerations for laserplasma linear colliders, Phys. Rev. ST Accel. Beams 13, 101301 (2010).

[9] W. P. Leemans et al., Multi-GeV Electron Beams from Capillary-Discharge-Guided Subpetawatt Laser Pulses in the Self-Trapping Regime, Phys. Rev. Lett. 113, 245002 (2014).

[10] X. Wang et al., Quasi-monoenergetic laser-plasma acceleration of electrons to $2 \mathrm{GeV}$, Nat. Commun. 4, 1988 (2013).

[11] C. E. Clayton, C. Joshi, C. Darrow, and D. Umstadter, Relativistic Plasma-Wave Excitation by Collinear Optical Mixing, Phys. Rev. Lett. 54, 2343 (1985).

[12] S. Y. Tochitsky, R. Narang, C. V. Filip, P. Musumeci, C. E. Clayton, R. B. Yoder, K. A. Marsh, J. B. Rosenzweig, C. Pellegrini, and C. Joshi, Experiments on laser driven beatwave acceleration in a ponderomotively formed plasma channel, Phys. Plasmas 11, 2875 (2004).

[13] C. B. Schroeder, E. Esarey, C. G. R. Geddes, Cs. Tóth, and W. P. Leemans, Design considerations for a laser-plasma linear collider, http://www.iaea.org/inis/collection/ NCLCollectionStore/_Public/41/103/41103142.pdf.

[14] C. B. Schroeder, E. Esarey, and W. P. Leemans, Beamstrahlung considerations in laser-plasma-accelerator-based linear colliders, Phys. Rev. ST Accel. Beams 15, 051301 (2012).

[15] I. V. Pogorelsky, I. Ben-Zvi, M. Babzien, Z. Najmudin, N. Dover, and W. Lu, New opportunities for strong-field LPI research in the mid-IR, Proc. SPIE Int. Soc. Opt. Eng. 9509, 95090P (2015).

[16] The Linear Collider Physics Resource Book for Snowmass 2001, http://www.slac.stanford.edu/th/LCBook/.

[17] I. Hinchliffte and M. Battaglia, A TeV linear collider, Phys. Today 57, No. 9, 49 (2004).

[18] I. V. Pogorelsky, Prospects for laser wakefield accelerators and colliders using $\mathrm{CO}_{2}$ laser drivers, Nucl. Instrum. Methods Phys. Res., Sect. A 410, 524 (1998).

[19] S. Gordienko and A. Pukhov, Scalings for ultrarelativistic laser plasmas and quasimonoenergetic electrons, Phys. Plasmas 12, 043109 (2005).

[20] B. Badelek et al., The photon collider at TESLA, Int. J. Mod. Phys. A 19, 5097 (2004). 
[21] V. Telnov, Principles of photon colliders, Nucl. Instrum. Methods Phys. Res., Sect. A 355, 3 (1995).

[22] I. V. Pogorelsky, Ultra-bright X-ray and gamma sources by Compton backscattering of $\mathrm{CO}_{2}$ laser beams, Nucl. Instrum. Methods Phys. Res., Sect. A 411, 172 (1998).

[23] C. B. Schroeder, E. Esarey, C. G. R. Geddes, C. Tóth, W. P. Leemans, C. B. Schroeder, W. Leemans, and E. Esarey, Design considerations for a laser-plasma linear collider, AIP Conf. Proc. 1086, 208 (2009).

[24] M. Battaglia, A. de Roeck, J. R. Ellis, and D. Schulte, CERN Report No. 2004-005, ISBN: 92-9083-226-6; M. Battaglia, A. de Roeck, J. R. Ellis, and D. Schulte, http:// cds.cern.ch/record/749219? ln=en.

[25] V.E. Balakin and A. A. Mikhailichenko, VLEPP; The conversion system for obtaining highly polarized electrons and positrons, in Proceedings of the 12th International Conference High-Energy Accelerators, 1983, edited by F. T. Cole and R. Donaldson, Vol. 127 (Fermi National Accelerator Laboratory, Batavia, IL, 1984), http:// www-project.slac.stanford.edu/lc/local/PolarizedPositrons/ doc/ClassicalPapers/balakin83.pdf.

[26] K. Abe et al., KEK Report No. 2001-11, arXiv:hep-ph/ 0109166 (2001) http://arxiv.org/pdf/hep-ph/0109166v1 .pdf.

[27] T. Hirose, K. Dobashi, Y. Kurihara, T. Muto, T. Omori, T. Okugi, I. Sakai, J. Urakawa, and M. Washio, Polarized positron source for the linear collider, JLC, Nucl. Instrum. Methods Phys. Res., Sect. A 455, 15 (2000).

[28] I. V. Pogorelsky et al., Demonstration of $8 \times 10^{18}$ photons/ second peaked at $1.8 \AA$ in a relativistic Thomson scattering experiment, Phys. Rev. ST Accel. Beams 3, 090702 (2000).

[29] J. Urakawa, Development of a compact X-ray source based on Compton scattering using a $1.3 \mathrm{GHz}$ superconducting RF accelerating linac and a new laser storage cavity, Nucl. Instrum. Methods Phys. Res., Sect. A 637, S47 (2011).

[30] W. S. Graves, W. Brown, F. X. Kaertner, and D. E. Moncton, MIT inverse Compton source concept, Nucl. Instrum. Methods Phys. Res., Sect. A 608, S103 (2009).

[31] V. Yakimenko and I. V. Pogorelsky, Polarized $\gamma$ source based on Compton backscattering in a laser cavity, Phys. Rev. ST Accel. Beams 9, 091001 (2006).

[32] A. Ovodenko et al., High duty cycle inverse compton scattering x-ray source, Appl. Phys. Lett. (2016).

[33] https://www.bnl.gov/atf/.

[34] I. V. Pogorelsky, M. Babzien, I. Ben-Zvi, J. Skaritka, and M. N. Polyanskiy, BESTIA - The next generation ultra-fast $\mathrm{CO}_{2}$ laser for advanced accelerator research, Nucl. Instrum. Methods Phys. Res., Sect. A 829, 432 (2016).

[35] A. J. Alcock and P. B. Corkum, Ultra-fast switching of infrared radiation by laser-produced carriers in semiconductors, Can. J. Phys. 57, 1280 (1979).

[36] C. V. Filip, R. Narang, S. Y. Tochitsky, C. E. Clayton, and C. Joshi, Optical Kerr switching technique for the production of a picosecond, multiwavelength $\mathrm{CO}_{2}$ laser pulse, Appl. Opt. 41, 3743 (2002).

[37] M. N. Polyanskiy and M. Babzien, Ultrashort Pulses, in $\mathrm{CO}_{2}$ Laser-Optimisation and Application, Vol. 139 (InTech, 2012).

[38] P. B. Corkum, Amplification of picosecond 10-m pulses in multiatmosphere $\mathrm{CO}_{2}$ lasers, IEEE J. Quantum Electron. QE-21, 216 (1985).

[39] S. Y. Tochitsky, C. Filip, R. Narang, C. E. Clayton, K. A. Marsh, and C. Joshi, Efficient shortening of self-chirped picosecond pulses in a high-power $\mathrm{CO}_{2}$ amplifier, Opt. Lett. 26, 813 (2001).

[40] M. N. Polyanskiy, I. V. Pogorelsky, and V. Yakimenko, Picosecond pulse amplification in isotopic $\mathrm{CO}_{2}$ active medium, Opt. Express 19, 7717 (2011).

[41] M. N. Polyanskiy, M. Babzien, and I. V. Pogorelsky, Chirped-pulse amplification in a $\mathrm{CO}_{2}$ laser, Optica 2, 675 (2015).

[42] M. N. Polyanskiy, U. S. Patent application No. 62/021725, 2014.

[43] E. P. Velikhov et al., Pulsed $\mathrm{CO}_{2}$ Laser and Their Application for Isotope Separation (Nauka, Moscow, 1983).

[44] H. Huang, M. Uder, R. Barthelmess, and J. Dorn, Application of high power thyristors in HVDC and FACTS systems, http://www.ptd.siemens.de/080731_Paper262_ cepsi08_valve_final.pdf.

[45] H. J. Schulze, F.-J. Niedernostheide, U. Kellner-Werdehausen, J. Przybilla, and M. Uder, High-voltage thyristors for HVDC and other applications: Light-triggering combined with selfprotection functions, http://www.infineon.com/dgdl/ Infineon_Bipolar-High_voltage_Thyristors_for_HVDC-EDv1.0-en.pdf?fileId=db3a304412b407950112b40ec0c61261.

[46] J. Choi, Introduction of the magnetic pulse compressor (MPC)-fundamental review and practical application, J. Electr. Eng. Tech. 5, 484 (2010).

[47] I. Smilanski, S. R. Byron, and T. R. Burkes, Electrical excitation of an $\mathrm{XeCl}$ laser using magnetic pulse compression, Appl. Phys. Lett. 40, 547 (1982).

[48] X. L. Xu, Y. P. Wu, C. J. Zhang, F. Li, Y. Wan, J. F. Hua, C.-H. Pai, W. Lu, P. Yu, C. Joshi, and W. B. Mori, Low emittance electron beam generation from a laser wakefield accelerator using two laser pulses with different wavelengths, Phys. Rev. ST Accel. Beams 17, 061301 (2014).

[49] C. B. Schroeder, J.-L. Vay, E. Esarey, S. S. Bulanov, C. Benedetti, L.-L. Yu, M. Chen, C. G. R. Geddes, and W. P. Leemans, Thermal emittance from ionization-induced trapping in plasma accelerators, Phys. Rev. ST Accel. Beams 17, 101301 (2014).

[50] I. V. Pogorelsky and I. Ben-Zvi, Brookhaven National Laboratory's Accelerator Test Facility: research highlights and plans, Plasma Phys. Controlled Fusion 56, 084017 (2014). 